\title{
ESTABILIDAD ROBUSTA EN UN SISTEMA MECÁNICO CONTROLABLE
}

\section{ROBUST STABILITY IN A CONTROLLABLE MECHANICAL SYSTEM}

\author{
Raúl Temoltzi-Ávila ${ }^{1}$, Roberto Ávila-Pozos ${ }^{2}$, Ricardo Cruz-Castillo ${ }^{3}$
}

\section{Resumen}

En este trabajo se estudia el problema sobre la estabilidad robusta de la planificación de trayectorias de un móvil con dos ruedas de tracción y una rueda de dirección. Se deduce el modelo que describe la cinemática y se aplica una trasformación conocida para proponer un control mediante el cual el móvil alcanza una trayectoria deseada. El control se elige como la velocidad angular de la rueda de dirección. Se discute el caso de alcanzar una trayectoria circular y se dan ejemplos numéricos para dicha trayectoria.

Palabras clave: estabilidad robusta, sistemas de control, perturbaciones permanentes

\section{Abstract}

In this paper we study the problem of the robust stability of the planning of trajectories of a mobile with two traction wheels and a steering wheel. The model describing the kinematics is derived and a known transformation is applied to propose a control by which the mobile reaches the desired path. The control is chosen as the angular speed of the steering wheel. The case of reaching a linear trajectory is discussed and numerical examples are given for that trajectory.

Keywords: robust stability, control systems, permanent disturbances

\footnotetext{
${ }^{1}$ Raúl Temoltzi-Ávila. Profesor Investigador del Área Académica de Matemáticas y Física. Doctor en Ciencias por la Benemérita Universidad Autónoma de Puebla, México. Sus temas de investigación están relacionados con la teoría de control y estabilidad de sistemas controlables. Autor de correspondencia. Correo-e: temoltzi@uaeh.edu.mx

${ }^{2}$ Roberto Ávila-Pozos. Profesor Investigador del Área Académica de Matemáticas y Física. Doctor en Ciencias por la Universidad Autónoma Metropolitana, México. Sus temas de investigación están relacionados con células excitables, epidemiología matemática y dinámica de poblaciones.

${ }^{3}$ Ricardo Cruz-Castillo. Profesor Investigador del Área Académica de Matemáticas y Física. Doctor en Ciencias por la Benemérita Universidad Autónoma de Puebla, México. Sus temas de investigación están relacionados con la teoría de control y estabilidad de sistemas controlables.
} 


\section{INTRODUCCIÓN}

Un problema importante en ingeniería consiste en la planificación de trayectorias de robots móviles autónomos. En la literatura este problema ha sido analizado en diferentes contextos. Por ejemplo, en Gregoria et al. (2012) se considera el problema de alcanzar trayectorias cerradas descritas por segmentos de rectas y círculos mediante un móvil con dos ruedas que giran de manera independiente y una tercera que gira libremente, en cuyo caso, la solución se obtiene de forma numérica. Un método distinto de resolver el problema de planificación de trayectorias se plantea en Gilimyanov et al. (2008), donde se consideran las ecuaciones cinemáticas de un móvil conformado por cuatro ruedas, de las cuales dos son fijas y giran a una velocidad constante, mientras que las dos restantes se emplean para obtener la dirección del móvil.

En este trabajo se describe un modelo cinemático clásico de un móvil con tres ruedas, al cual se le aplica la transformación descrita en Gilimyanov et al. (2008). Tomando como base lo anterior, se presenta el problema sobre la estabilidad robusta en la planificación de trayectorias en el modelo cinemático.

\section{MODELO CINEMÁTICO Y SU TRANSFORMACIÓN}

Se considera un móvil con dos ruedas de tracción trasera que son impulsadas por un motor a una velocidad constante, y una rueda delantera de dirección que permite conducir el móvil hasta una trayectoria $\mathbf{p}(s)$ regular y parametrizada por su longitud de arco, la cual es llamada trayectoria deseada. Se supone que el móvil no se encuentra sobre dicha trayectoria, por lo que se requiere buscar alguna estrategia que permita conducirlo hasta ella, y una vez logrado este objetivo, se desea que permanezca sobre la trayectoria o en una vecindad de la misma.

Las ecuaciones que describen la cinemática se consideran en un marco de referencia inercial $(\xi, \eta)$, mientras que el móvil se considera como un cuerpo rígido en el que se fija un sistema de referencia no inercial $\left(\xi^{\prime}, \eta^{\prime}\right)$. La trayectoria que el móvil describe, denotada por $\mathbf{r}(t)$, se observa desde un punto $P$ ubicado en el origen del sistema de referencia no inercial, como se observa en la Figura 1. Las posiciones de las ruedas traseras derecha e izquierda en el sistema $\left(\xi^{\prime}, \eta^{\prime}\right)$ tienen coordenadas $\mathbf{r}_{r}=(0, a)^{\top}$ y $\mathbf{r}_{l}=(0,-a)^{\top}$, las coordenadas de la rueda de dirección son $\mathbf{r}_{f}=(L, 0)^{\top}$, el vector velocidad del móvil es $\mathbf{v}=\left(v_{\xi^{\prime}}, v_{\eta^{\prime}}\right)^{\top}$ y su rapidez $v=\|\mathbf{v}\|$. Dado que las componentes de la velocidad del punto $P$ con respecto al sistema inercial están determinadas por $v \cos (\alpha)$

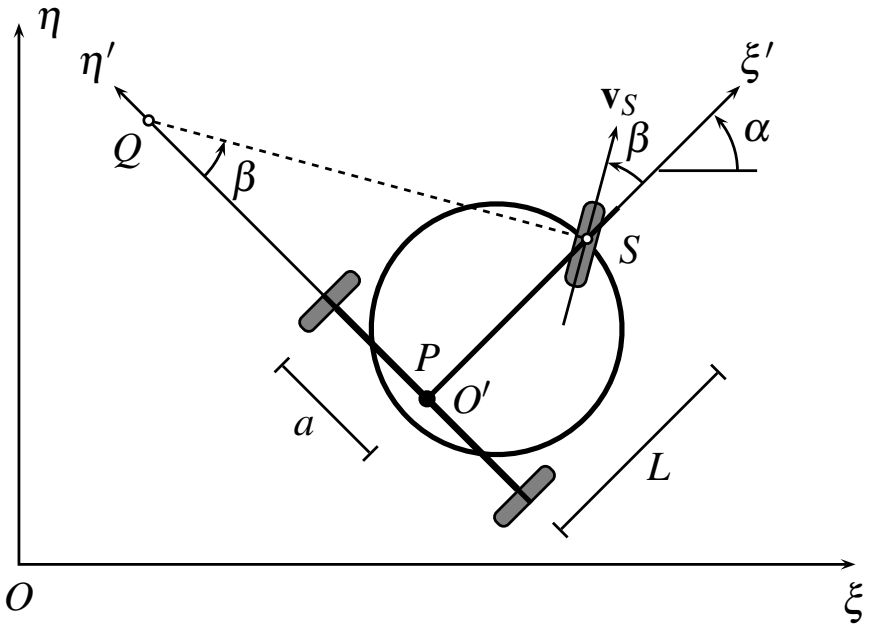

Figura 1: Esquema del móvil. y $v \operatorname{sen}(\alpha)$, estas describen la velocidad neta del punto $P$, es decir,

$$
\dot{\xi}=v \cos (\alpha), \quad \dot{\eta}=v \operatorname{sen}(\alpha) .
$$

El sistema (1) depende del ángulo $\alpha$ que define la dirección de la rueda delantera $\mathbf{r}_{f}$ por medio del ángulo $\beta=\beta(t)$. La relación para $\beta$ y la velocidad angular $\omega$ de la rueda delantera $\mathbf{r}_{f}$ es $\dot{\beta}=\omega$, la cual es medida en $\left(\xi^{\prime}, \eta^{\prime}\right)$. Por otra parte, si $Q$ denota el centro instantáneo de curvatura (es decir, 
el punto que resulta de interceptar la recta ortogonal al eje de la rueda trasera y la recta ortogonal al eje de la rueda delantera), $R_{P}=\overline{P Q}$ el radio de curvatura y $\bar{u}=R_{P}^{-1}$ la curvatura de la trayectoria del móvil, entonces resulta $\tan (\beta)=\bar{u} L$, lo cual se observa del triángulo $Q P S$. Esta relación muestra la dependencia $\bar{u}=\bar{u}(\beta)$. Si las coordenadas del punto $P$ en el sistema de referencia inercial son $(\xi, \eta)$, y las coordenadas del centro de la rueda delantera son $\left(\xi_{2}, \eta_{2}\right)$, entonces resulta $\xi_{2}=\xi+L \cos (\alpha)$ y $\eta_{2}=\eta+L \operatorname{sen}(\alpha)$. Así, la velocidad $\mathbf{v}_{S}$ del punto $\left(\xi_{2}, \eta_{2}\right)$ tiene componentes $\dot{\xi}_{2}=v \cos (\alpha)-L \dot{\alpha} \operatorname{sen}(\alpha)$ y $\dot{\eta}_{2}=v \operatorname{sen}(\alpha)+L \dot{\alpha} \cos (\alpha)$. Si el vector velocidad $\mathbf{v}_{S}$ se rota un ángulo $\alpha+\beta$ en sentido de las agujas de reloj, resulta que la segunda componente del nuevo vector es cero respecto al sistema de referencia inercial $(\xi, \eta)$, es decir

$$
-\operatorname{sen}(\alpha+\beta)(v \cos (\alpha)-L \dot{\alpha} \operatorname{sen}(\alpha))+\cos (\alpha+\beta)(v \operatorname{sen}(\alpha)+L \dot{\alpha}, \cos (\alpha))=0
$$

debido a que el móvil no tiene desplazamiento sobre el eje $\eta^{\prime}$, y en este caso coincide con el eje $\eta$. Se observa entonces que esta ecuación equivale a $v \operatorname{sen}(\beta)-L \cos (\beta) \dot{\alpha}=0$, de donde $L \dot{\alpha}=v \tan (\beta)$.

Por tanto, las ecuaciones que describen la cinemática del móvil son

$$
\begin{aligned}
\dot{\xi} & =v \cos (\alpha), \\
\dot{\eta} & =v \operatorname{sen}(\alpha), \\
\dot{\alpha} & =v \bar{u}(\beta), \\
\dot{\beta} & =\omega,
\end{aligned}
$$

donde $\bar{u}(\beta)=L^{-1} \tan (\beta)$. Con el fin de estudiar el sistema no lineal (2) se emplea la transformación descrita en Gilimyanov et al. (2008), la cual permite asociar un sistema lineal que es más simple de analizar. La transformación considera el intercambio de las derivadas de la variable temporal $t$, denotadas por $\left({ }^{*}\right)$, por las derivadas con respecto a la derivada de $r=r(t)=\int_{0}^{t}\|\dot{\mathbf{r}}(\tau)\| d \tau$, denotadas por $\left(^{\prime}\right)$.

Se supone que $P$ es el punto que describe la dinámica del móvil; $A$ el punto que se encuentra en la trayectoria deseada $\mathbf{p}(s)$ más cercano a $P$ y $Q$ el centro de curvatura instantánea de $\mathbf{p}(s)$. La velocidad instantánea del móvil en el punto $P$ se obtiene de la relación $\mathbf{v}(t)=\dot{\mathbf{r}}(t)$ y su magnitud es $v=v(t)=\|\mathbf{v}(t)\|$; en tal caso $\dot{r}=v$. La distancia entre los puntos $A \mathrm{y}$ $Q$ se denota por $\overline{A Q}=R_{A}(s)=[k(s)]^{-1}$, donde $k=k(s)$ es la curvatura de la trayectoria $\mathbf{p}(s)$ en el punto $A ; \tau$ el ángulo comprendido entre la línea tangente a la trayectoria $\mathbf{p}(s)$ en $A$ y el eje horizontal $\eta=0 ; \alpha$ es

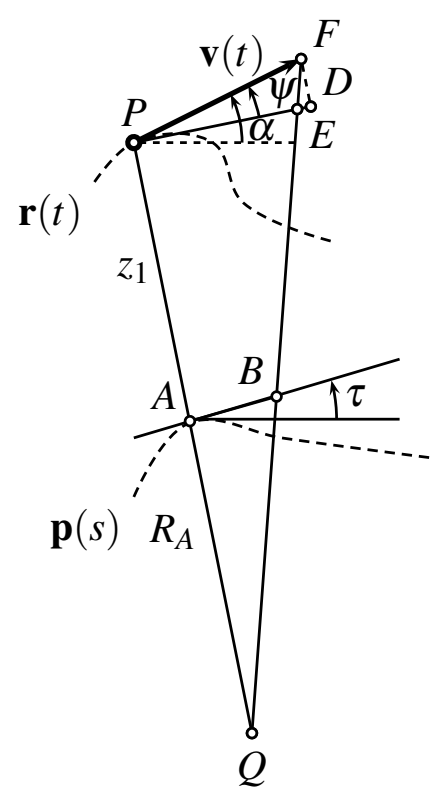

Figura 2: Esquema del cambio de variable. el ángulo descrito por la recta tangente a la trayectoria $\mathbf{r}(t)$ en el punto $P$ con respecto al eje horizontal y $\psi=\alpha-\tau$.

Cuando el móvil alcanza la trayectoria deseada $\mathbf{p}(s)$ y permanece sobre ella, resulta que $\psi=0$, ya que en ese caso el segmento $\overline{A B}$ coincide con $\overline{P F}$.

Se introduce la variable $z_{1}$ definida como la distancia $\overline{A P}$ y se observa que cuando $z_{1}$ varía, el ángulo $\psi$ también lo hace. Se puede mostrar que a partir del sistema (2) se obtiene el sistema

$$
\begin{aligned}
& z_{1}^{\prime}=z_{2} \\
& z_{2}^{\prime}=z_{3} \\
& z_{3}^{\prime}= \pm \sqrt{1-z_{2}^{2}}\left(L \bar{u}^{2}+\frac{1}{L}\right) \frac{\omega}{v}-f(\mathbf{z})
\end{aligned}
$$


donde

$$
f(\mathbf{z})=\frac{z_{2} z_{3}^{2}}{1-z_{2}^{2}}-\frac{k z_{2} z_{3}}{1+k z_{1}}-\frac{k^{2} z_{2}\left(1-z_{2}^{2}\right)}{\left(1+k z_{1}\right)^{2}} \pm \frac{k_{s}^{\prime} \sqrt{\left(1-z_{2}^{2}\right)^{3}}}{\left(1+k z_{1}\right)^{3}}
$$

$\mathbf{z}=\left(z_{1}, z_{2}, z_{3}\right)^{\top} \in \mathbb{R}^{3}$, y $k_{s}^{\prime}$ satisface la relación $k^{\prime}=k_{s}^{\prime} s^{\prime}$. Se observa que la variable $z_{2}$ esta acotada: $-1 \leq z_{2} \leq 1$. Los detalles de la transformación se pueden consultar en Gilimyanov et al. (2008).

Dependiendo de la forma en la que se elija la curvatura de la trayectoria deseada y la aceleración angular en la rueda delantera del móvil, el sistema (1) describirá el comportamiento del sistema. En el caso descrito se elige la velocidad angular de igual manera a como se hace en Gilimyanov et al. (2008):

$$
\omega= \pm \frac{L v(f(\mathbf{z})-q(\mathbf{z})+u)}{\sqrt{1-z_{2}^{2}}\left(L^{2} \bar{u}^{2}+1\right)}
$$

donde $q(\mathbf{z})=\mathbf{a}^{\top} \mathbf{z}$ con $\mathbf{a}=\left(a_{1}, a_{2}, a_{3}\right) \in \mathbb{R}^{3}$ constante, $u(\cdot) \in \mathscr{U}=\left\{u \in L_{\infty}:|u(t)| \leq \delta_{1}\right\}$ es una función escalar que representa una perturbación externa para el móvil y $L_{\infty}$ denota el conjunto de funciones acotadas casi en todas partes. Después de sustituir esta expresión se obtiene el sistema asociado a (3):

$$
\begin{aligned}
& z_{1}^{\prime}=z_{2} \\
& z_{2}^{\prime}=z_{3} \\
& z_{3}^{\prime}=-a_{3} z_{1}-a_{2} z_{2}-a_{1} z_{3}+u,
\end{aligned} \quad u(\cdot) \in \mathscr{U} .
$$

La estabilidad de la solución trivial del sistema (4) determina la forma bajo la cual el móvil alcanza la trayectoria deseada.

\section{ESTABILIDAD ROBUSTA DEL SISTEMA ASOCIADO}

Cuando en el sistema (4) se considera $u(\cdot) \equiv 0$, en Gilimyanov et al. (2008) se muestra que la elección de (9) como un control para el sistema (3), siempre que se elija de forma adecuada el vector constante $\mathbf{a} \in \mathbb{R}^{3}$, permite conducir el móvil hasta la trayectoria deseada de forma asintótica, toda vez que el problema se traduce en estabilizar de forma asintótica la solución de este sistema, es decir, tal que la condición $\lim _{t \rightarrow+\infty}\|\mathbf{z}(t)\|=0$ sea satisfecha. No obstante, si se supone que el sistema (2) admite perturbaciones externas (lo cual equivale a suponer que $u(\cdot) \not \equiv 0$ ), entonces la estabilidad asintótica de la solución trivial $\mathbf{z} \equiv \mathbf{0}$ del sistema (4) puede no tener cabida, en tal caso el concepto sobre la estabilidad bajo perturbaciones permanentes, cuya definición fue introducida por I. G. Malkin y G. N. Duboshin, puede ser empleada (ver por ejemplo (Elsgoltz, 1969, pag. 240)). Se considera así la siguiente:

Definición 1. La solución trivial $\mathbf{z} \equiv \mathbf{0}$ del sistema (4) es robustamente estable, si dado $\varepsilon>0$ existen $\delta_{1}=\delta_{1}(\varepsilon)>0$ y $\delta_{2}=\delta_{2}(\varepsilon)>0$ tales que $|u(t)| \leq \delta_{1}$ y $\|\mathbf{z}(0)\|<\delta_{2}$ implican que $\|\mathbf{z}(t)\| \leq \varepsilon$ para todo $t>0$ y toda perturbación externa $u(\cdot) \in \mathscr{U}$.

Se supone que la norma introducida en la Definición 1 es $\|\mathbf{z}\|=\operatorname{máx}\left\{\left|z_{1}\right|,\left|z_{2}\right|,\left|z_{3}\right|\right\}$.

Siguiendo el desarrollo de Alexandrov et al. (2015) se observa que si los coeficientes en el sistema (4) se expresan como $a_{1}=\varepsilon_{1}+2 \varepsilon_{2}, a_{2}=2 \varepsilon_{1} \varepsilon_{2}+\varepsilon_{2}^{2}+\omega_{2}^{2}$ y $a_{3}=\varepsilon_{1}\left(\varepsilon_{2}^{2}+\omega_{2}^{2}\right)$, con $\varepsilon_{1}, \varepsilon_{2}, \omega_{2}>0$, entonces al emplear la transformación $z_{1}=\left(\varepsilon_{2}^{2}+\omega_{2}^{2}\right) \rho^{-1} \tilde{z}_{1}+2 \varepsilon_{2} \rho^{-1} \tilde{z}_{2}+\rho^{-1} \tilde{z}_{3}, z_{2}=\varepsilon_{1} \tilde{z}_{1}+\tilde{z}_{2}$ y $z_{3}=\varepsilon_{1} \tilde{z}_{2}+\tilde{z}_{3}$, donde $\rho=\left(\varepsilon_{2}-\varepsilon_{1}\right)^{2}+\omega_{2}^{2}$, el sistema (4) es equivalente al siguiente par de sistemas desacoplados

$$
\dot{z}_{1}=-\varepsilon_{1} z_{1}+\rho^{-1} u, \quad u(\cdot) \in \mathscr{U},
$$




$$
\begin{aligned}
& \dot{z}_{2}=z_{3}, \\
& \dot{z}_{3}=-\left(\varepsilon_{2}^{2}+\omega_{2}^{2}\right) z_{2}-2 \varepsilon_{2} z_{3}+u, \quad u(\cdot) \in \mathscr{U},
\end{aligned}
$$

Respecto a este nuevo sistema se puede realizar el análisis sobre la estabilidad robusta en el sentido de la Definición 1 al considerar el problema de determinar las perturbaciones externas $u(\cdot) \in \mathscr{U}$ que proporcionen valores extremos a los funcionales $\varphi_{i}[\tilde{\mathbf{z}}(t)]=\left|\tilde{z}_{i}(t)\right|, i=1,2,3$, y para los cuales se plantean los problemas de optimización:

$$
\varphi_{i}[\tilde{\mathbf{z}}(t)] \rightarrow \sup _{u(\cdot) \in \mathscr{U}}, \quad i=1,2,3 .
$$

Los valores que se obtienen son llamados desviaciones máximas del sistema (5a)-(5b) y las funciones $u^{o}(\cdot) \in \mathscr{U}$ que permiten obtener esos valores son llamadas peores perturbaciones. Las desviaciones máximas así como las peores perturbaciones para los subsistemas dados son conocidas y son como sigue, de acuerdo a Alexandrov et al. (2015): la peor pertubación del primer sistema es la función constante $u^{o}(t)=\delta_{1}$ y la desviación máxima es $\alpha_{1}^{*}=\delta_{1} \alpha_{1}$, con

$$
\alpha_{1}=\rho^{-1} \varepsilon_{1}^{-1}
$$

y para el segundo sistema la peor perturbación es $u^{o}(t)=\delta_{1} \operatorname{sign}\left(\tilde{z}_{3}(t)\right)$ y las desviaciones máximas toman la forma $\alpha_{2}^{*}=\delta_{1} \alpha_{2}$ y $\alpha_{3}^{*}=\delta_{1} \alpha_{3}$, con

$$
\alpha_{2}=\frac{1}{\varepsilon_{2}^{2}+\omega_{2}^{2}} \operatorname{coth}\left(\frac{\pi \varepsilon_{2}}{2 \omega_{2}}\right), \quad \alpha_{3}=\frac{1}{\sqrt{\varepsilon_{2}^{2}+\omega_{2}^{2}}}\left(1+\operatorname{coth}\left(\frac{\pi \varepsilon_{2}}{2 \omega_{2}}\right)\right) e^{-\frac{\varepsilon_{2}}{\omega_{2}}\left(\frac{\pi}{2}-\arctan \frac{\varepsilon_{2}}{\omega_{2}}\right)} .
$$

De esta manera, si en los sistemas (5a)-(5b) se consideran las condiciones iniciales tal que $\left|\tilde{z}_{k}^{0}\right| \leq \alpha_{k}^{*}, k=1,2,3$, entonces las coordenadas de las soluciones cumplen las desigualdades $\left|\tilde{z}_{k}(t)\right| \leq \alpha_{k}^{*}$ para toda perturbación externa $u(\cdot) \in \mathscr{U}$ y $t \geq 0$. Por tanto, se puede construir un paralelepípedo $\mathrm{P}_{\tilde{\mathbf{z}}}$ en $\mathbb{R}^{3}$ cuyos vértices $\left\{\delta_{1} \tilde{\mathbf{z}}_{1}, \delta_{1} \tilde{\mathbf{z}}_{2}, \ldots, \delta_{1} \tilde{\mathbf{z}}_{8}\right\}$ dependen de las desviaciones máximas de los sistemas (5a)-(5b), donde $\tilde{\mathbf{z}}_{1}=\left(\alpha_{1}, \alpha_{2}, \alpha_{3}\right)^{\top}, \tilde{\mathbf{z}}_{2}=\left(\alpha_{1},-\alpha_{2}, \alpha_{3}\right)^{\top}, \ldots, \quad \tilde{\mathbf{z}}_{8}=\left(\alpha_{1},-\alpha_{2},-\alpha_{3}\right)^{\top}$, y tales que $\tilde{\mathbf{z}}(t) \in \mathrm{P}_{\tilde{\mathbf{z}}}$ para todo $t \geq 0$, debido a que

$$
\|\tilde{\mathbf{z}}(t)\| \leq \delta_{1} \cdot \operatorname{máx}_{1 \leq k \leq 8}\left\|\tilde{\mathbf{z}}_{k}\right\| .
$$

El estimado anterior es válido para toda solución de (5a)-(5b) obtenida con cada $u(\cdot) \in \mathscr{U}$, lo cual muestra que las soluciones son acotadas para las condiciones iniciales dadas. Es conocido de Alexandrov et al. (2015) que al considerar la peor perturbación $u^{o}(t)=\delta_{1} \operatorname{sign}\left(\tilde{z}_{3}(t)\right)$, las coordenadas $\left\{\tilde{z}_{2}(t), \tilde{z}_{3}(t)\right\}$ de la solución $\tilde{\mathbf{z}}(t)$, tienden a un único ciclo límite

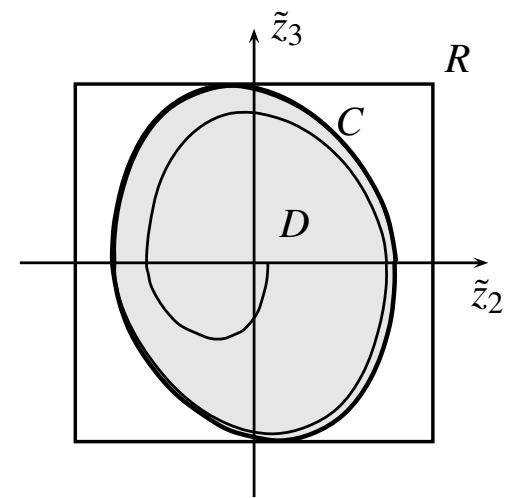

Figura 3: Representación de las desviaciones máximas y los conjuntos que de ellas se generan. máximo orbitalmente asintóticamente estable $C$, el cual intersecta de forma tangencial al menos dos caras de $\mathrm{P}_{\tilde{\mathbf{x}}}$ en una sección transversal $R$ (ver la Figura 3), y cuyas ecuaciones paramétricas son:

$$
\begin{aligned}
& \tilde{z}_{2}(t)=\mp\left(\frac{\delta_{1}}{\varepsilon_{2}^{2}+\omega_{2}^{2}}+\alpha_{2}^{*}\right) \cdot e^{-\varepsilon_{2} t}\left(\cos \left(\omega_{2} t\right)+\frac{\varepsilon_{2}}{\omega_{2}} \sin \left(\omega_{2} t\right)\right) \pm \frac{\delta_{1}}{\varepsilon_{2}^{2}+\omega_{2}^{2}} \\
& \tilde{z}_{3}(t)= \pm \frac{\varepsilon_{2}^{2}+\omega_{2}^{2}}{\omega_{2}}\left(\frac{\delta_{1}}{\varepsilon_{2}^{2}+\omega_{2}^{2}}+\alpha_{2}^{*}\right) e^{-\varepsilon_{2} t} \sin \left(\omega_{2} t\right)
\end{aligned}
$$


donde $0 \leq t \leq \frac{\pi}{\omega_{j}}$. El ciclo límite máximo $C$ coincide con la frontera del conjunto de alcanzabilidad $D$ de la ecuación diferencial con una perturbación externa:

$$
\ddot{\tilde{z}}_{2}+2 \varepsilon_{2} \dot{\tilde{z}}_{2}+\left(\varepsilon_{2}^{2}+\omega_{2}^{2}\right) \tilde{z}_{2}=u, u(\cdot) \in \mathscr{U}, \quad\left(\tilde{z}_{2}(0), \dot{\tilde{z}}_{2}(0)\right) \in D
$$

Se concluye que las soluciones de (5a)-(5b) son contenidas propiamente en la estimación del conjunto de alcanzabilidad $\mathrm{C}_{\tilde{\mathbf{z}}}=\left[-\alpha_{1}^{*}, \alpha_{1}^{*}\right] \times D$, el cual es un cilindro que posee la propiedad $\mathrm{C}_{\tilde{\mathbf{z}}} \subset \mathrm{P}_{\tilde{\mathbf{z}}}$.

La estabilidad robusta de la solución trivial $\mathbf{z} \equiv \mathbf{0}$ del sistema (4) para perturbaciones externas, se puede obtener de considerar el estimado de calidad robusta para los sistemas (5a)-(5b): para $\varepsilon>0$ dado, el estimado de $\|\mathbf{z}(t)\| \leq \varepsilon$ para todo $0 \leq t<+\infty$ y toda perturbación externa $u(\cdot) \in \mathscr{U}$, se obtiene de tomar $\delta_{1}=\varepsilon / \chi$, donde

$$
\chi=\frac{\varepsilon}{\delta_{1}(\varepsilon)}=\operatorname{máx}_{1 \leq k \leq 8}\left\|S \tilde{\mathbf{z}}_{k}\right\|
$$

siempre que la condición inicial del sistema satisfaga la desigualdad

$$
\|\mathbf{z}(0)\| \leq \delta_{2}:=\delta_{1}(\varepsilon) \cdot \operatorname{máx}_{1 \leq k \leq 8}\left\|S \tilde{\mathbf{z}}_{k}\right\|
$$

Debido a la linealidad de la transformación empleada, se sigue que la solución del sistema (4) satisface las relaciones $\mathbf{z}(t) \in \mathrm{C}_{\mathbf{z}} \subset \mathrm{P}_{\mathbf{z}}$, donde $\mathrm{C}_{\mathbf{z}}=S \mathrm{C}_{\tilde{\mathbf{z}}}$ y $\mathrm{P}_{\mathbf{z}}=S \mathrm{P}_{\tilde{\mathbf{z}}}$. Debe advertirse que la peor perturbación que proporciona las desviaciones máximas para el sistema (4) se obtiene de la transformación $\mathbf{z}=S \tilde{\mathbf{z}}$, la cual es dada por $u^{o}(t)=\delta_{1} \operatorname{sign}\left(\varepsilon_{1} z_{2}(t)+z_{3}(t)\right)$.

\section{SIMULACIÓN DE LA PLANIFICACIÓN DE TRAYECTORIAS}

Se supone en este apartado que las trayectorias que el móvil debe alcanzar son esencialmente curvas regulares. La elección de las parametrizaciones de tales curvas $\mathbf{p}:[0, T] \rightarrow \mathbb{R}^{2}$ se elige mediante su longitud de arco $s=\int_{0}^{s}\|\dot{\mathbf{p}}(t)\| d t$ para cada $s \in[0, T]$, es decir, tales que $\|\dot{\mathbf{p}}(s)\|=1$ para todo $s \in[0, T]$. Es conocido que toda curva regular posee un parámetro definido por su longitud de arco.

Como resumen de los resultados sobre la estabilidad robusta de los sistema de orden tres, puede realizarse una simulación de la dinámica del móvil, cuyo sistema es

$$
\begin{array}{ll}
\dot{\xi}=v \cos (\alpha), & \xi(0)=\xi_{0}, \\
\dot{\eta}=v \sin (\alpha), & \eta(0)=\eta_{0}, \\
\dot{\alpha}=v L^{-1} \tan (\beta(t)), & \alpha(0)=\alpha_{0},
\end{array}
$$

donde $\beta(t)$ satisface la ecuación integral para una constante $\beta_{0} \in\left[-\beta_{\text {máx }}, \beta_{\text {máx }}\right]$

$$
\beta(t)=\beta_{0} \pm L v \int_{0}^{t} \frac{f(\mathbf{z}(s))-q(\mathbf{z}(s))+u^{o}(s)}{\sqrt{1-z_{2}^{2}(s)}} \cos ^{2}(\beta(s)) d s
$$

la cual se obtiene de sustituir (3) en la cuarta ecuación de (2), y donde $\mathbf{z}(t)=\left(z_{1}(t), z_{2}(t), z_{3}(t)\right)^{\top}$ es la solución del sistema (4), la cual depende de la peor perturbación $u^{o}(t)=\delta_{1} \operatorname{sign}\left(\varepsilon_{1} z_{2}(t)+z_{3}(t)\right)$. En general, el problema de obtener solución a la ecuación integral (9) no es fácil de resolver, pero puede obtenerse de forma numérica.

El siguiente ejemplo muestra el problema sobre la estabilidad robusta en el problema de planificar una trayectoria circular con centro en $(0,0)$ y radio $r>0$; en tal caso, su parametrización por longitud de arco es $\mathbf{p}(s)=\left(r \cos \left(r^{-1} s\right), r \sin \left(r^{-1} s\right)\right)$ y su curvatura es $k=\left\|\mathbf{p}^{\prime \prime}(s)\right\|=r^{-1}$. 

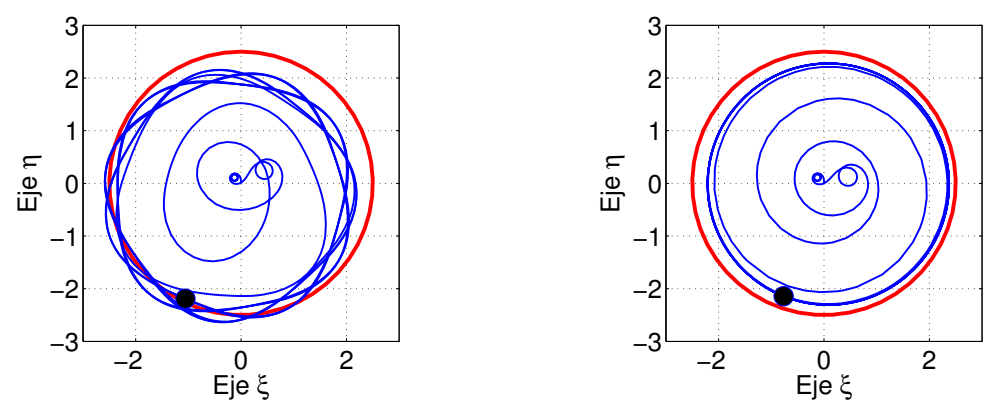

Figura 4: Estabilidad robusta en la planificación de trayectorias

Ejemplo. Se considera el problema de alcanzar un circulo de radio $r=2.5$ unidades, partiendo desde la posición inicial $\left(\xi_{0}, \eta_{0}\right)=(0,0)$. Se considera un móvil de longitud $L=0.1$ unidades que se mueve a una velocidad constante $v=5$ unidades por segundo. Si se espera que la influencia de la peor perturbación provoque comportamientos no deseados en el móvil al alcanzar la trayectoria deseada, se elige $u^{o}(t)=\delta_{1} \operatorname{sign}\left(\varepsilon_{1} z_{2}(t)+z_{3}(t)\right)$ y la condición inicial $\mathbf{z}(0)=(0.7,0,0)^{\top}$. En la Figura 4 se muestra el comportamiento con la elección $\varepsilon_{1}=1, \varepsilon_{2}=5$ y $\omega_{2}=8$ tomando valores $\varepsilon=0.1$ y $\varepsilon=0.001$ para garantizar el nivel de convergencia hacia la trayectoria deseada.

\section{CONCLUSIÓN}

Las condiciones obtenidas respecto a la estabilidad robusta en el problema sobre la planificación de trayectorias en el modelo descrito, muestran la posibilidad de alcanzar trayectorias con cierto margen de error considerando el problema de desviación máxima en sistemas lineales de orden tres. En el ejemplo presentado se ha considerado únicamente el caso de una trayectoria de curvatura constante, no obstante el mismo procedimiento puede emplearse en el caso de curvaturas no constantes.

\section{REFERENCIAS}

Alexandrov, V. V., Sánchez-Flores, L., and Temoltzi-Avila, R. (2015). Una nota sobre la estabilidad robusta en sistemas de ecuaciones diferenciales oscilatorias con perturbaciones externas. PÄDI. Boletín Científico de Ciencias Básicas e Ingeniería del ICBI, 3(5):1-11.

Elsgoltz, L. (1969). Ecuaciones diferenciales y cálculo variacional. Mir, Moscú.

Gilimyanov, R. F., Pesterev, A. V., and Rapoport, L. B. (2008). Motion control for a wheeled robot following a curvilinear path. Journal of Computer and Systems Sciences International, 47(6):987994.

Gregoria, C. M., Alexandrov, V. V., and Moises, G. A. (2012). Dynamic model of a mobile robot with two active wheels and the design an optimal control for stabilization. IEEE, 2012:219-224. 